\begin{tabular}{|c|c|c|c|}
\hline Caco Donorto & Case Rep Gastroenterol 2019 & & \\
\hline troenterology & $\begin{array}{l}\text { DOI: 10.1159/000504695 } \\
\text { Published online: December 10, } 2019\end{array}$ & $\begin{array}{l}\text { (c) } 2019 \text { The Author(s) } \\
\text { Published by S. Karger AG, Basel } \\
\text { www.karger.com/crg }\end{array}$ & $\begin{array}{l}\text { Karger } \\
\text { Open'access }\end{array}$ \\
\hline & $\begin{array}{l}\text { This article is licensed under the } \\
\text { International License (CC BY-NC) (h } \\
\text { Usage and distribution for commercial }\end{array}$ & $\begin{array}{l}\text { nons Attribution-NonCommercia } \\
\text { ger.com/Services/OpenAccessLic } \\
\text { uires written permission. }\end{array}$ & \\
\hline
\end{tabular}

\title{
Retroperitoneal Liposarcoma with Multilocular Cysts
}

\author{
Chika Komine $^{a} \quad$ Minoru Fukuchi $^{a}$ Shinji Sakurai $^{b}$ Yuichi Tabe $^{a}$ \\ Akihiko Sano $^{a}$ Kana Saito $^{a}$ Takaharu Fukasawa $^{a}$ Hideki Yamamoto $^{a}$ \\ Masachika Tani $^{a}$ Hiroshi Naitoh $^{a}$ Hiroshi Saeki $^{c} K_{\text {Ken Shirabe }}^{c}$ \\ aDepartment of Surgery, Japan Community Health Care Organization, Gunma Chuo \\ Hospital, Maebashi, Japan; 'bepartment of Diagnostic Pathology, Japan Community \\ Health Care Organization, Gunma Chuo Hospital, Maebashi, Japan; 'Department of \\ General Surgical Science, Gunma University Graduate School of Medicine, \\ Maebashi, Japan
}

\section{Keywords}

Liposarcoma $\cdot$ Retroperitoneum $\cdot$ Multilocular cysts

\begin{abstract}
In this study, we describe a 60-year-old man with a giant retroperitoneal liposarcoma with multilocular cysts. He was admitted to our hospital because of a 5-month history of abdominal distention. Abdominal computed tomography revealed a giant lobulated cystic mass occupying the retroperitoneal space that contained partially solid fat components. Magnetic resonance imaging indicated that this complex mass exhibited a low signal intensity on a T1weighted image, whereas it exhibited a high and focally intermediate signal intensity on a T2weighted image. This patient was diagnosed with a mucinous type of retroperitoneal sarcoma, which was then resected. During surgery, the tumor was isolated from the retroperitoneum and other organs, but the detachment was required only because of fixation around the left external iliac artery. The histological diagnosis was a well-differentiated liposarcoma with multilocular cysts that contained old bloody, serous, and mucinous fluids, which are a rare phenomenon in liposarcoma. This case indicates that retroperitoneal liposarcoma should also be considered as a differential diagnosis of retroperitoneal cystic mass.
\end{abstract}




\section{Introduction}

Liposarcoma is one of the most common sarcomas of the retroperitoneum. This tumor is a soft tissue sarcoma of adipocyte origin and can be further divided into 3 groups: (1) welldifferentiated/dedifferentiated liposarcoma (WDL/DL); (2) myxoid/round cell liposarcoma; and (3) pleomorphic liposarcoma. WDL/DL is a major subtype of liposarcoma that contains a solid component and has an anatomical predilection for the retroperitoneum [1]. The presence of macroscopic fat is characteristic of a diagnosis of liposarcoma. However, several intraperitoneal or retroperitoneal liposarcoma cases may show cystic change as a certain component [2-5].

Limited to the retroperitoneum, only 3 cases of liposarcoma with cystic changes have been reported [3-5]. Here, we report a case of retroperitoneal WDL with multilocular cysts that was treated by surgical resection.

\section{Case Report}

A 60-year-old man was admitted to our hospital because of a 5-month history of abdominal distention without any previous injury to the area. The patient had a history of appendectomy for acute appendicitis. Blood test results showed elevated levels of creatinine $(1.14 \mathrm{mg} / \mathrm{dL})$. Abdominal computed tomography (CT) revealed a giant lobulated cystic mass occupying the retroperitoneal space and containing partially solid fat components with calcification in the left portion (Fig. 1a, b). The exclusion of bilateral ureter due to the mass exhibited hydronephrosis (Fig. 1c). Magnetic resonance imaging (MRI) revealed a complex mass that exhibited a low intensity signal on a T1-weighted image, whereas the mass exhibited a high and focally intermediate signal intensity on a T2-weighted image (Fig. 1d, e). 18F-fluorodeoxyglucose-positron emission tomography (FDG-PET) imaging showed a slight accumulation of FDG in the solid components (data not shown).

The patient first underwent insertion of ureteral stents for the treatment of bilateral hydronephrosis. We radiologically diagnosed a mucinous type of retroperitoneal sarcoma, and because we suspected a liposarcoma, tumor resection was then performed. During surgery, it was discovered that the tumor occupied the entire abdominal cavity but was present mainly in the left side of the abdomen. The tumor was isolated from the retroperitoneum and other organs, but detachment was required only because of fixation around the left external iliac artery. The resected tumor measured approximately $23 \times 21 \times 15 \mathrm{~cm}$ and weighed $4 \mathrm{~kg}$.

Macroscopically, the tumor was encapsulated by a smooth connective fibrous capsule (Fig. 2a). The cut surface of the tumor showed a multicystic and yellowish lipomatous mass that contained old bloody, serous, and mucinous fluids (Fig. 2b). Histologically, the tumor consisted of mature fat, atypical lipoblasts, and mesenchymal cells (Fig. 3a), but no epithelial component was observed on the surface of the cyst wall (Fig. 3b). Immunohistochemically, mature fat and some atypical mesenchymal tumor cells were positive for S-100 protein (Fig. 3c) and diffusely positive for p16 (Fig. 3d). Based on these histological and immunohistochemical results, the tumor was diagnosed as WDL with multilocular cysts. Microscopically, the surgical margin around the left external iliac artery was positive.

The postoperative course was uneventful, and the patient was discharged 14 days after surgery and underwent removal of the ureteral stents 42 days after surgery. Postoperatively, the patient received a total of 56 Gy of intensity-modulated radiation therapy (IMRT) around the left external iliac artery with a positive surgical margin. He exhibited local recurrence 
surrounding the left adrenal gland 7 months after the first surgery. Therefore, he underwent a second surgery, which included a left adrenalectomy. The histological results of the resected specimens were indicative of mixed WDL/DL, and the surgical margin was also positive. He then received a total of 54 Gy of IMRT around the portion of the left adrenalectomy. He remained alive for 17 months from the first treatment and was then followed up with no indication of recurrence.

\section{Discussion}

Liposarcoma is the most common primary retroperitoneal sarcoma and accounts for $40 \%$ of all cases [6]. Most sarcomas cannot be characterized by a specific cell type based on imaging, but liposarcoma is an exception. Imaging modalities, such as CT or MRI, may help in the diagnosis of liposarcoma. In WDLs, characteristic CT findings include macroscopic fat comprising $>75 \%$ of the tumor with smooth margins and lobular contours [7]. MRI demonstrates high signal intensity in T1-weighted images and intermediate signal intensity in T2-weighted images corresponding to the fatty areas, with loss of signal in fat-suppressed images [8]. In DLs, characteristic CT or MRI findings include a nonlipomatous mass within, adjacent to, or surrounding a fatty mass $[9,10]$. However, if these tumors are primarily composed of a solid and cystic mass, the differentiation of liposarcomas from other sarcoma types becomes difficult $[11,12]$. To our knowledge, 3 other cases of cystic retroperitoneal WDL/DL have been previously described [3-5]. Our present case highlights a rare retroperitoneal WDL with multilocular cysts.

The differential diagnoses of retroperitoneal cystic mass are divergent and include neoplastic or nonneoplastic lesions, such as mature teratoma, mucinous cystadenoma, lymphangioma, epidermoid cyst, and hematoma [11]. In our case, a retroperitoneal cystic mass was radiologically diagnosed as a mucinous type of retroperitoneal liposarcoma because partially solid fat was confirmed in the cystic mass. Of the 3 other previously reported cases of cystic retroperitoneal liposarcomas, 2 masses were radiologically diagnosed each as a cystic renal cell carcinoma and a simple cyst, respectively $[3,5]$, while the third was diagnosed as a highgrade sarcoma by biopsy [4]. Based on these case reports, an accurate diagnosis of cystic retroperitoneal liposarcoma by imaging or biopsy might be difficult. As a result, all 4 cases, which were suspected malignancies that were diagnosed as large retroperitoneal cystic masses, were treated by surgical resection [3-5].

In this case, histopathological and immunohistochemical examination showed that the tumor was correctly diagnosed as a retroperitoneal WDL with multilocular cysts. The other 3 case reports suggest that histopathological cystic changes may be due to necrosis, lysis, hemorrhage, or ischemia of the tumor [3-5]. Since the tumor in our case had a fibrous cystic wall without an epithelial component and contained old bloody, serous, and mucinous fluids, the cystic changes may have been due to hemorrhage or ischemia. However, the histopathology underlying the cystic changes remains unclear.

The treatment of retroperitoneal liposarcoma is complete surgical resection of the tumor, even if the tumor presents with cystic changes [12]. This case had a positive surgical margin, and the patient experienced local recurrence 7 months after the first surgery. Of the 3 cases of cystic retroperitoneal liposarcomas, 2 had negative surgical margins and no recurrence 6 months and 10 months after surgery [3,5], while 1 case had residual tumor after surgery following chemotherapy and radiotherapy (RT) [4]. Negative surgical margins offer the possibility of longer overall and recurrence-free survival [13]. However, retroperitoneal liposarcoma, 
which frequently recurs within 6 months to 2 years after the first surgery, infiltrates adjacent tissues and structures with skip areas, despite surgical resection with negative margins [12]. Therefore, the propensity of retroperitoneal liposarcoma demands complementary adjuvant or neoadjuvant therapy.

The value of adjuvant or neoadjuvant chemotherapy for the treatment of retroperitoneal liposarcoma remains controversial [12]. Recent studies have reported that adjuvant or neoadjuvant RT for retroperitoneal sarcoma or liposarcoma is associated with improvements in overall survival and decreases in local recurrence compared with surgery alone [14, 15]. As adequate radiation doses are administered without a concomitant to normal tissue increase in toxicity, refined delivery methods, such as IMRT, 3-dimensional RT, helical tomotherapy, proton or heavy ion RT, and stereotactic RT, have been used recently. IMRT has been found to yield superior treatment plans with respect to overall radiation dose conformality and good tolerance [16]. We thus performed surgery and administered adjuvant IMRT twice for primary and recurrent retroperitoneal liposarcoma.

In summary, we reported in this study a rare case of retroperitoneal liposarcoma with multilocular cysts, which is valuable in terms of the differential diagnosis of a retroperitoneal cystic mass. Surgery is the primary treatment, but the role of adjuvant therapy is widely debated.

\section{Acknowledgement}

The authors would like to thank the staff of Gunma Chuo Hospital for help.

\section{Statement of Ethics}

The patient gave his informed consent before publication.

\section{Disclosure Statement}

The authors have no conflicts of interest to declare.

\section{Funding Sources}

This study did not receive any funding support.

\section{Author Contributions}

C. Komine, M. Fukuchi, S. Sakurai, Y. Tabe, A. Sano, K. Saito, T. Fukasawa, H. Yamamoto, M. Tani, and H. Naitoh interpreted our patient's imaging and discussed a treatment plan. C. Komine, M. Fukuchi, and K. Saito performed surgery on the patient. S. Sakurai conducted the pathological analysis. The manuscript was prepared by C. Komine under the supervision of M. Fukuchi, H. Naitoh, H. Saeki, and K. Shirabe. All authors approved the final version of the manuscript. 


\section{References}

1 Goldblum JR, Weiss SW, Folpe AL. Liposarcoma. In: Goldblum J, Weiss S, Folpe AL. Enziger and Weiss's soft tissue tumors. 6th ed. Philadelphia: Elsevier Health Sciences; 2013. pp. 484-523.

2 Grifasi C, Calogero A, Carlomagno N, Campione S, D'Armiento FP, Renda A. Intraperitoneal dedifferentiated liposarcoma showing MDM2 amplification: case report. World J Surg Oncol. 2013 Nov;11(1):305.

3 Horiguchi A, Oyama M. [Perinephric liposarcoma mimicking cystic renal tumor]. Nippon Hinyokika Gakkai Zasshi. 2002 Mar;93(3):491-4.

4 Khoury M, Sim GC, Harao M, Radvanyi L, Amini B, Benjamin RS, et al. Multicystic dedifferentiated retroperitoneal liposarcoma: tumour cyst fluid analysis and implications for management. BMJ Case Rep. 2015 Jul;2015.

5 Uchihashi K, Matsuyama A, Shiba E, Kimura Y, Ogata T, Yabuki K, et al. Retroperitoneal dedifferentiated liposarcoma with huge cystic degeneration: A case report. Pathol Int. 2017 May;67(5):264-8.

6 Cormier JN, Pollock RE. Soft tissue sarcomas. CA Cancer J Clin. 2004 Mar-Apr;54(2):94-109.

7 Chang IY, Herts BR. Retroperitoneal liposarcoma. J Urol. 2013 Mar;189(3):1093-4.

8 Elsayes KM, Staveteig PT, Narra VR, Chen ZM, Moustafa YL, Brown J. Retroperitoneal masses: magnetic resonance imaging findings with pathologic correlation. Curr Probl Diagn Radiol. 2007 May-Jun;36(3):97106.

9 Kim T, Murakami T, Oi H, Tsuda K, Matsushita M, Tomoda K, et al. CT and MR imaging of abdominal liposarcoma. AJR Am J Roentgenol. 1996 Apr;166(4):829-33.

10 Kumarasamy NA, Gayer G. Retroperitoneal sarcomas. Semin Ultrasound CT MR. 2011 Oct;32(5):422-32.

11 Scali EP, Chandler TM, Heffernan EJ, Coyle J, Harris AC, Chang SD. Primary retroperitoneal masses: what is the differential diagnosis? Abdom Imaging. 2015 Aug;40(6):1887-903.

12 Vijay A, Ram L. Retroperitoneal liposarcoma: a comprehensive review. Am J Clin Oncol. 2015 Apr;38(2):2139.

13 Lewis JJ, Leung D, Woodruff JM, Brennan MF. Retroperitoneal soft-tissue sarcoma: analysis of 500 patients treated and followed at a single institution. Ann Surg. 1998 Sep;228(3):355-65.

14 Nussbaum DP, Rushing CN, Lane WO, Cardona DM, Kirsch DG, Peterson BL, et al. Preoperative or postoperative radiotherapy versus surgery alone for retroperitoneal sarcoma: a case-control, propensity score-matched analysis of a nationwide clinical oncology database. Lancet Oncol. 2016 Jul;17(7):966-75.

15 Lee HS, Yu JI, Lim DH, Kim SJ. Retroperitoneal liposarcoma: the role of adjuvant radiation therapy and the prognostic factors. Radiat Oncol J. 2016 Sep;34(3):216-22.

16 Paumier A, Le Péchoux C, Beaudré A, Negretti L, Ferreira I, Roberti E, et al. IMRT or conformal radiotherapy for adjuvant treatment of retroperitoneal sarcoma? Radiother Oncol. 2011 Apr;99(1):73-8. 


\section{Case Reports in Gastroenterology}

\begin{tabular}{l|l}
\hline Case Rep Gastroenterol 2019;13:514-520 \\
\hline DOI: 10.1159/000504695 & $\begin{array}{l}\text { @ 2019 The Author(s). Published by S. Karger AG, Basel } \\
\text { www.karger.com/crg }\end{array}$ \\
\hline
\end{tabular}

Komine et al.: Retroperitoneal Liposarcoma with Multilocular Cysts
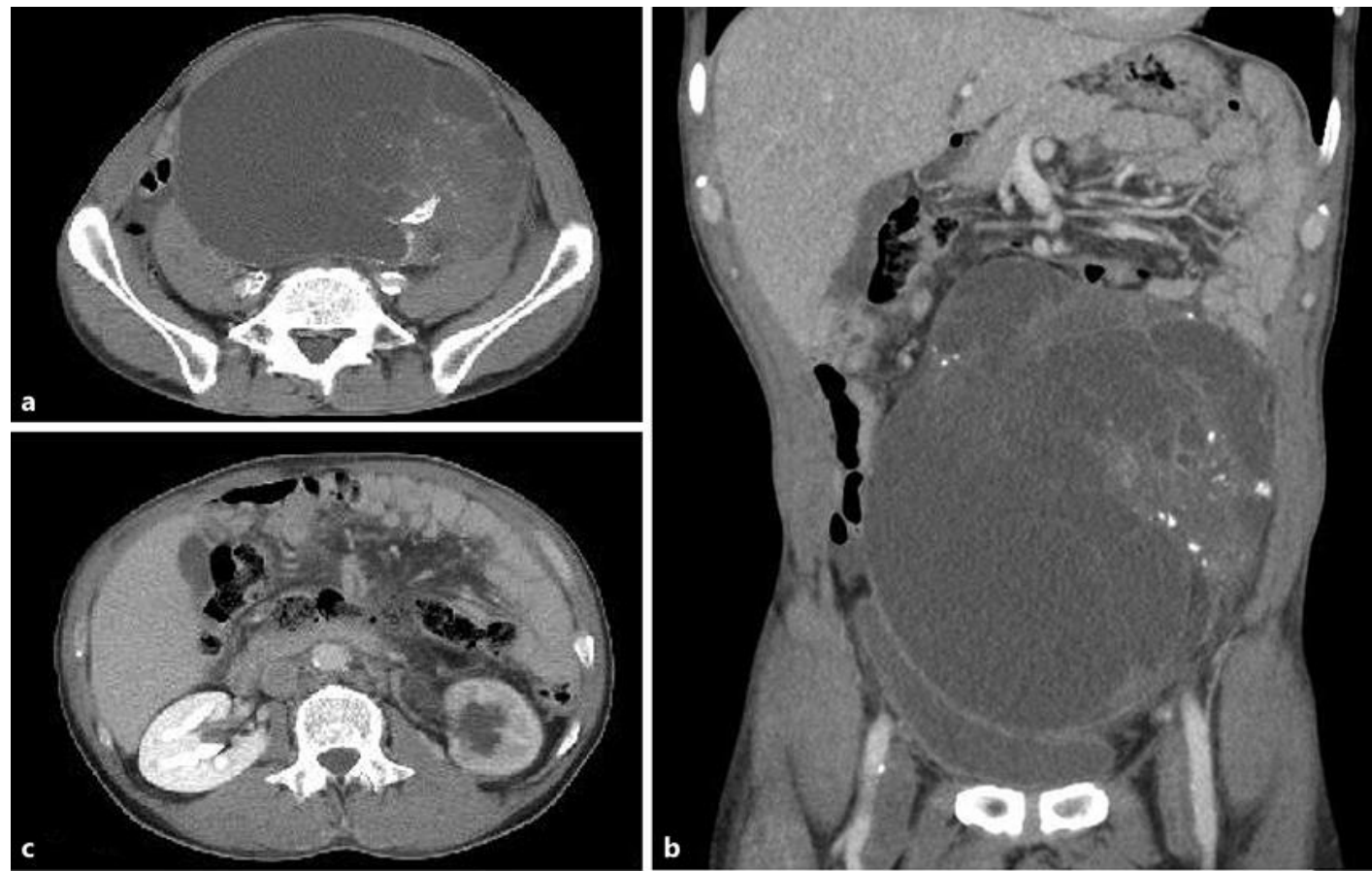

b
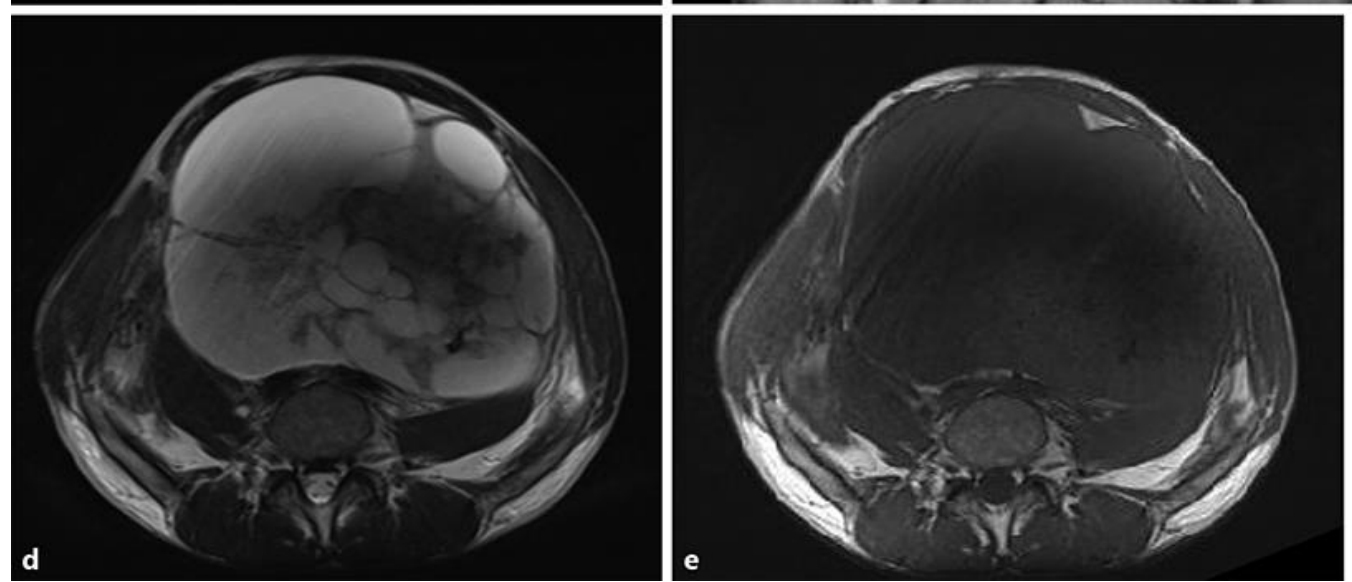

Fig. 1. CT images (a, c, axial; b, coronal). a, b A giant lobulated cystic mass that occupied the retroperitoneal space was observed and included partially solid fat components with calcification in the left portion. $\mathbf{c}$ Exclusion of the mass exhibited bilateral hydronephrosis. MRI images (axial). $\mathbf{d}$ The retroperitoneal mass demonstrated a low intensity on a T1-weighted image. e The lobulated mass demonstrated a high and focally intermediate intensity on a T2-weighted image. 

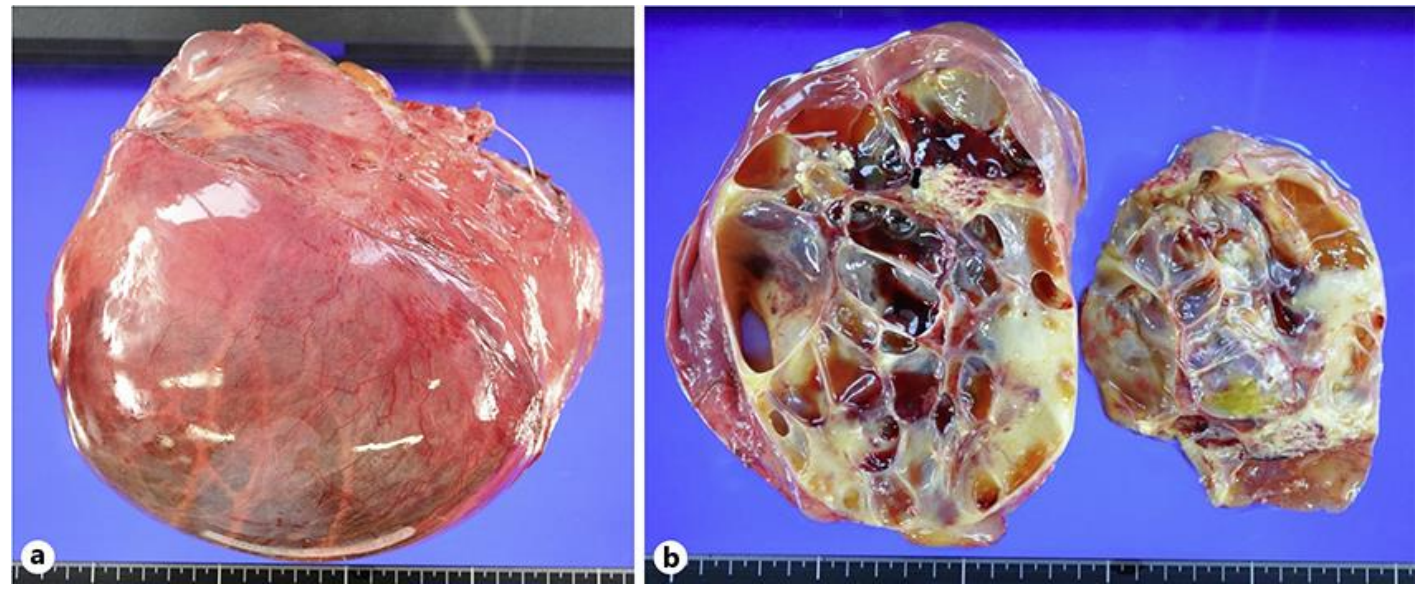

Fig. 2. Macroscopic examination. a The whole tumor was encapsulated by a smooth connective fibrous capsule. $\mathbf{b}$ The cut surface of the tumor was composed of a multicystic and yellowish lipomatous mass that included old bloody, serous, and mucinous fluids.

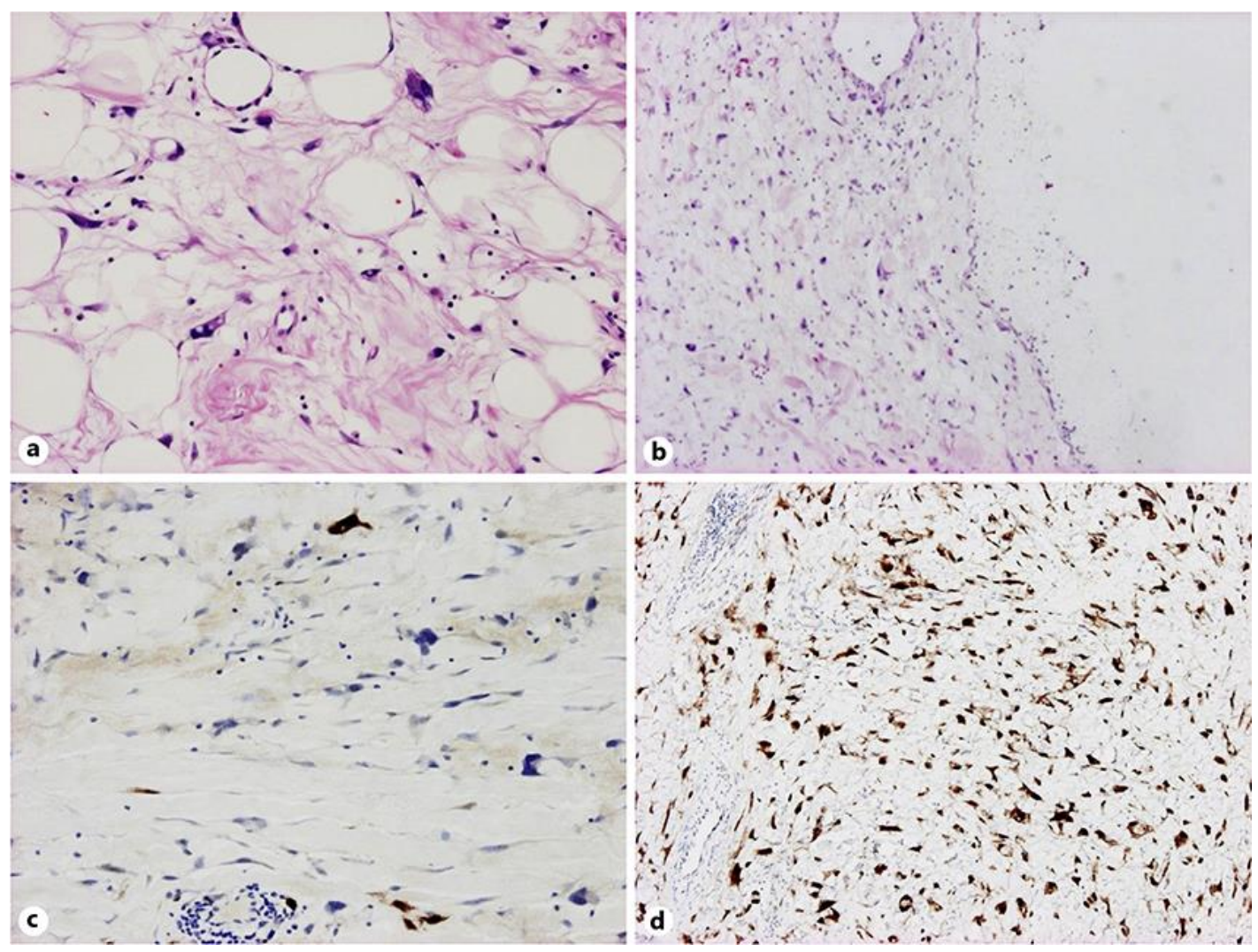

Fig. 3. Microscopic examination. a The tumor consisted of mature fat, atypical lipoblasts, and mesenchymal cells. $\mathbf{b}$ The cyst wall was not composed of epithelial cells. $\mathbf{c}$ Immunostaining for S-100 shows positive tumor cells. d Immunostaining for p16 shows that the tumor cells were diffusely positive. 